\title{
Effects of PUFA supplementation evidenced by brain imaging
}

Basant K. PURI

Department of Imaging,

Hammersmith Hospital,

Du Cane Road,

London W12 OHS, UK

$<$ basant.puri@imperial.ac.uk>

\begin{abstract}
This paper describes how the effects of PUFA supplementation can be indexed by neuroimaging. The role of structural magnetic resonance imaging studies are detailed in respect of testing a brain (lipid) hypothesis and in respect of using a gold-standard image registration technique. The role of magnetic resonance spectroscopy of the brain is considered with respect to a recent advance in the analysis of 31-phosphorus neurospectroscopy data that enables motion-restricted membrane phospholipids to be quantified.
\end{abstract}

Key words: image registration, membrane phospholipids, MRI, neurospectroscopy, PUFA supplementation

supplementation (Behan et al., 1990)). Unfortunately structural brain MRI changes were not assessed during this randomized, double-blind, placebo-controlled trial, but subsequently a pilot study has indicated that eicosapentaenoic acidrich PUFA supplementation in myalgic encephalomyelitis may be associated with beneficial brain changes, with a reduction in the size of the lateral ventricles (Puri et al., 2004). The question of whether, at baseline, myalgic encephalomyelitis is associated with changes in brain structure compared with unaffected age- and gender-matched controls has been addressed by a number of studies; the most recent and largest voxel-based morphometry study of 26 patients and 26 matched controls has shown that this disease appears to be associated with reduced grey matter in the occipital lobes, right angular gyrus and left parahippocampal gyrus, and reduced white matter in the left occipital lobe (Puri et al., 2011).

\section{Gold-standard registration}

In Euclidean three-space $\left(E^{3}\right)$, monomodal rigid body registration is clearly associated with six parameters (three translational and three rotational degrees of freedom). There are two steps involved in the registration, namely the registration itself and transformation via resampling (one image being transformed according to the estimated parameters) (Friston, 1997; Puri, 2004). The resampling requires image interpolation, and here there are three main methods available: simple interpolation (nearest neighbour - zero-order hold or trilinear first-order hold); generalized interpolation (B-spline or o-Mom (maximal-order interpolation of minimal support) basis functions); and windowed sinc interpolation (Friston, 1997, 2007). It is the last of these which is the gold-standard for rigid body transformation, being a method involving convolving the image with a sinc function centred on a point to be re-sampled, and that gives results relatively close to a Fourier interpolation in $\mathbb{R}^{3}$ (Puri, 2004; Friston, 2007). Since the sinc function tends to infinity, to reduce the computational expense of the associated calculations it is appropriate to truncate the function by using a Hanning window. Also, only a limited number of nearest neighbours are usually sampled, rather than every image voxel. Using this method, several studies have shown that supplementation with the semi-synthetic omega-3 PUFA deriving from post-viral fatigue syndrome, gave positive results in favour of the PUFA 
ative ethyl-eicosapentaenoic acid is associated with lateral ventricular shrinkage in disorders as diverse as schizophrenia, treatment-resistant depression and Huntington's chorea (or Huntington's disease) (Puri et al., 2000, 2001, 2002).

\section{1-phosphorus neurospectroscopy}

The way in which brain cell membrane phospholipid metabolism changes in association with PUFA supplementation is of academic and clinical importance. During the last decade of the twentieth century, the main method used noninvasively to study human brain cell membrane phospholipids in vivo was via 31-phosphorus magnetic resonance spectroscopy and the quantification of membrane phospholipid metabolism carried out through the measurement of phosphomonoesters, which index membrane phospholipid anabolism, and phosphodiesters, which index membrane phospholipid catabolism. Unfortunately, this methodology only provides an indirect measure of membrane phospholipids.

It is now possible non-invasively to study brain cell membrane motion-restricted phospholipids directly, in spite of the large chemical-shift anisotropy of the 31-phosphorus-containing moieties that are confined to a relatively rigid (in nuclear magnetic resonance terms) membranous structure (Puri et al., 2008)). The method involves analysis of the broad spectral component which underlies the narrow peaks which are more usually the focus of attention of 31phosphorus neurospectroscopy data analysis. Indeed, it is interesting to note that in the past this very same broad component has actually been considered by some to be an unwanted spectral component which should be removed (Estilaei et al., 2001). The broad component integral can be derived using the convolution difference resolution enhancement method from imageselected in vivo spectroscopy sequence spectra (Estilaei et al., 2001; Roth and Kimber, 1982). The required broad component is given by the difference between the measured raw signal intensity, $S$, and the term $S(1-f \cdot \exp (-\pi L t))$, where $f$ is the convolution-difference factor (that is, the fraction of the broad component contributing to the acquired signal at a given echo time), $L$ is an exponential filter, and $t$ represents time. The corresponding integral can be obtained by fitting the spectrum to multiple Gaussian lines, minimizing residuals, and summating over all Gaussian-line integrals. Estilaei and colleagues obtained $\mathrm{T}_{2}$-magnetization decay curves by plotting the broad component area as a function of the echo time (Estilaei et al., 2001). A suitable fit is given by $S(t)=S_{1} e^{-C}+S_{S} e^{-D}$, where $S(t)$ is the total magnetization at $t$, and $S_{1}$ and $S_{S}$ are the respective equilibrium magnetizations for the slow and fast decaying components, $C=$ (echo time)/(spin-spin relaxation time for the long $T_{2}$ component), and $D=$ (echo time) $/$ (spin-spin relaxation time for the short $\mathrm{T}_{2}$ component). With spin-spin relaxation time for the long $T_{2}$ component $\left(\mathrm{T}_{2 l}\right)$ » spin-spin relaxation time for the short $T_{2}$ component $\left(T_{2 s}\right)$ (Kilby et al., 1990), and with echo time $(T E) \approx T_{2 l}$, it can be demonstrated that In $S(T E)=\ln \left(S_{I}+S_{S}\right)-S_{S} T E /\left(T_{2 s}\left(S_{I}+S_{S}\right)\right)$, while with $T E \approx T_{2 s}$, the right-hand side of the last equation becomes approximately In $S_{1}-\mathrm{TE} / \mathrm{T}_{21}$ (Estilaei et al., 2001). The required corresponding fitting can then be simply carried out using linear registration.

Our group have analyzed the broad component in two different groups of patients with schizophrenia, one group of whom had committed serious and dangerous acts of violence while psychotic (including homicide) and the other group of whom had no forensic history and were suffering predominantly from negative symptoms, but so far we have been unable to find evidence consistent with the lipid membrane hypothesis of schizophrenia (Puri et al., 2008).

Clearly this new methodology should be used in future studies of PUFA supplementation.

Acknowledgements. I should like to thank the British Medical Research Council (MRC) for funding my work mentioned in this paper, and I should also like to thank Hammersmith Hospital, London and Imperial College London.

\section{REFERENCES}

Behan PO, Behan WM, Horrobin D. Effect of high doses of essential fatty acids on the postviral fatigue syndrome. Acta Neurol Scand 1990; 82: 209-16.
Estilaei MR, Matson GB, Payne GS, et al. Effects of chronic alcohol consumption on the broad phospholipid signal in human brain: an in vivo 31P MRS study. Alcohol Clin Exp Res 2001; 25: 89-97.

Friston KJ. Analysing brain image: principles and overview. In: Frackowiak RSJ, Friston KJ, Frith CD, Dolan RJ, Mazziotta JC (Eds.), Human brain function. London: Academic Press, 1997: 25-41.

Friston K. Statistical parametric mapping In: Friston KJ, Ashburner JT, Kiebel SJ, Nichols TE, Penny W D (Eds.), Statistical parametric mapping: The analysis of functional brain images. London: Academic Press, 2007: 10-31.

Horrobin DF. Post-viral fatigue syndrome, viral infections in atopic eczema, and essential fatty acids. Med Hypotheses 1990; 32: 211-7.

Kilby PM, Allis JL, Radda GK. Spin-spin relaxation of the phosphodiester resonance in the 31P NMR spectrum of human brain. The determination of the concentrations of phosphodiester components. FEBS Lett 1990; 272: 163-5.

Puri BK, Richardson AJ, Horrobin DF, et al. Eicosapentaenoic acid treatment in schizophrenia associated with symptom remission, normalisation of blood fatty acids, reduced neuronal membrane phospholipid turnover and structural brain changes. Int J Clin Pract 2000; 54: 57-63.

Puri BK, Counsell SJ, Hamilton G, Richardson AJ, Horrobin DF. Eicosapentaenoic acid in treatment-resistant depression associated with symptom remission, structural brain changes and reduced neuronal phospholipid turnover. Int J Clin Pract 2001; 55: 560-3.

Puri BK, Bydder GM, Counsell SJ, et al. MRI and neuropsychological improvement in Huntington disease following ethyl-EPA treatment. Neuroreport 2002; 13: 123-6.

Puri BK. Monomodal rigid-body registration and applications to the investigation of the effects of eicosapentaenoic acid intervention in neuropsychiatric disorders. Prostaglandins Leukot Essent Fatty Acids 2004; 71: 177-9.

Puri BK, Holmes J, Hamilton G. Eicosapentaenoic acid-rich essential fatty acid supplementation in chronic fatigue syndrome associated with symptom remission and structural brain changes. Int / Clin Pract 2004; 58: 297-9.

Puri BK, Counsell SJ, Hamilton G, Bustos MG, Treasaden IH. Brain cell membrane motionrestricted phospholipids in patients with schizophrenia who have seriously and dangerously violently offended. Prog Neuropsychopharmacol Biol Psychiatry 2008; 32: 751-4.

Puri BK, Counsell SJ, Hamilton G. Brain cell membrane motion-restricted phospholipids: A cerebral 31-phosphorus magnetic resonance spectroscopy study of patients with 
schizophrenia. Prostaglandins Leukot Essent Fatty Acids 2008; 79: 233-5.

Puri BK, Jakeman PM, Agour $\mathrm{M}$, et al. Regional grey and white matter volumetric changes in myalgic encephalomyelitis (chronic fatigue syndrome): A voxel-based morphometry 3T MRI study. Br J Radiol 2011 (in press).
Roth K, Kimber BJ. Determination of optimal parameters in the convolution difference resolution enhancement technique. Org Mag Reson 1982; 18: 197-8. 